\title{
Optimal strategies in repeated games with incomplete information: the dependent case
}

\author{
Fabien Gensbittel, Miquel Oliu-Barton ${ }^{\dagger}$
}

\begin{abstract}
Using the duality techniques introduced by De Meyer (1996a, 1996b), De Meyer and Marino (2005) provided optimal strategies for both players in finitely repeated games with incomplete information on two sides, in the independent case. In this note, we extend both the duality techniques and the construction of optimal strategies to the case of general type dependence.
\end{abstract}

\section{Contents}

1 Introduction $\quad 2$

2 Duality techniques $\quad 3$

2.1 Incomplete information on one side . . . . . . . . . . . . . . . 3

2.2 Incomplete information on two sides . . . . . . . . . . . . . 5

3 Repeated games with incomplete information $\quad 6$

3.1 Recursive structure . . . . . . . . . . . . . . . . . 7

3.2 The dual game . . . . . . . . . . . . . . . . . . . . . 9

3.3 Main result . . . . . . . . . . . . . . . . . . . . . . 10

3.4 Markovian optimal strategies . . . . . . . . . . . . . . . . . . 14

3.5 The independent case . . . . . . . . . . . . . . . . . . . . 14

4 Extensions, open problems $\quad 15$

4.1 Infinite set of actions . . . . . . . . . . . . . . . . 15

4.2 Optimal strategies in differential games with incomplete information . . . . . . 15

5 Acknowledgements $\quad 16$

6 Appendix 16

*TSE (GREMAQ, Université Toulouse 1 Capitole), France, email: fabien.gensbittel@tse-fr.eu

†PSL, Université Paris-Dauphine, Ceremade, 75016 Paris, France; email: miquel.oliu.barton@normalesup.org 


\section{Introduction}

We consider here a zero-sum repeated game with incomplete information on both sides, in the spirit of Aumann and Maschler [1]. Let $K$ (resp. L) be the finite set of types of Player 1 (resp. 2), and let $\pi$ be a probability distribution over $K \times L$. To any pair $(k, \ell)$ corresponds a matrix game $G^{k \ell}: I \times J \rightarrow \mathbb{R}$, where $I$ (resp. $J$ ) is the finite set of actions of Player 1 (resp. 2). The game is played as follows. First, a pair $(k, \ell) \in K \times L$ is drawn according to $\pi$. Player 1 (resp. 2) is informed only of $k$ (resp. $\ell$ ). Then, the game $G^{k \ell}$ is played repeatedly. At each stage $m \geq 1$, the players choose actions $\left(i_{m}, j_{m}\right) \in I \times J$ and produce a stage-payoff $G^{k \ell}\left(i_{m}, j_{m}\right)$ for Player 1 . Actions are publicly observed after each stage. To the realized stream of payoffs $\left(G^{k \ell}\left(i_{m}, j_{m}\right)\right)_{m \geq 1}$, we associate the $\theta$-weighted sum $\sum_{m \geq 1} \theta_{m} G^{k \ell}\left(i_{m}, j_{m}\right)$ where $\theta$ is a probability over the set of positive integers specifying the weight of each stage. For any initial distribution $\pi$ and evaluation $\theta$, we consider the game $\mathcal{G}_{\theta}(\pi)$ where the payoff of Player 1 is the expected $\theta$-weighted sum of the stage-payoffs. Particular choices of $\theta$ lead to the usual $n$-stage and $\lambda$-discounted games. The game has a value denoted $v_{\theta}(\pi)$ (see e.g. Lemma 3.2 below).

This model was analyzed by Mertens and Zamir in [8]. Their main result was the existence of a limit value for the $n$-stage repeated games as $n$ goes to $+\infty$ and its characterization as the unique solution of a system of functional equations. The proof of this result was based on the introduction of specific notion of concavity, called $I$-concavity, for the value function $v_{n}$. Precisely, any probability $\pi$ can be decomposed as a pair $(p, Q)$ where $p$ is a probability over $K$ and $Q$ is a transition probability from $K$ to $L$. Then, one may consider $v_{\theta}$ as a function of $(p, Q)$ and this function is concave with respect to $p$. A dual notion of II-convexity was also introduced and the notions of $I$-concave and II-convex envelopes were the building blocks of the system of functional equations characterizing the limit value. Based on this characterization, a construction of asymptotically optimal strategies (i.e. strategies being almost optimal in $\mathcal{G}_{\theta}(\pi)$, with an error term vanishing as the weights of each stage tend to 0) was obtained by Heuer [7] for $n$-stage games.

In addition to their main result, Mertens and Zamir [8] also established a recursive formula for $v_{\theta}$. However, the formula does not allow in general to construct recursively optimal strategies for the players, except in the case of incomplete information on one side (i.e. when $L$ is reduced to one point), where it can be used to construct optimal strategies for the informed player only. The notion of dual game was introduced by De Meyer in $[4,5]$ in order to provide a recursive construction of optimal strategies for the uninformed player in the case of games with incomplete information on one side. In contrast with the result of Heuer, the construction of De Meyer leads to optimal strategies in a game with fixed length and is not an asymptotic result. These duality techniques were later extended by De Meyer and Marino [6] for repeated games with incomplete information on both sides, in the special case of independent initial probabilities, i.e. $\pi=p \otimes q$, for some probabilities $p$ on $K$ and $q$ on $L$.

The construction of the dual game given in [6] for the independent case is related to the Fenchel conjugate of the function $p \rightarrow v_{\theta}(p \otimes q)$ for some fixed probability $q$ on $L$. In this note, using the notion of $I$-concavity and II-convexity, we extend the construction of the dual game for general, possibly dependent, initial probabilities $\pi$, by considering the notion of Fenchel conjugate of $I$-concave and II-convex functions. The same approach was taken by the second author in [9] to prove the existence and give a characterization of the value function for differential games with incomplete information. Our main result here is a recursive formula for the value function 
of the dual game from which we deduce a recursive manner to construct optimal strategies in the game $\mathcal{G}_{\theta}(\pi)$.

The most surprising aspect of this result is the need of adding an extra weight parameter to the dual game in order to obtain a recursive formula, which contrasts with the analogue results in the independent case [6].

\section{Duality techniques}

In this section, we will start by briefly recalling the construction and some results of [11, Chapter 2 ] for games with incomplete information on one side. Next, we establish analogue results for games with incomplete information on both sides. In all the games introduced below (primal and dual games), the model is known by both players and player 1 maximizes.

Notation 2.1. For a non-empty finite set $X, \Delta(X)$ denotes the set of probabilities over $X$, and is identified with the canonical simplex in $\mathbb{R}^{X} . \mathbb{N}^{*}$ denotes the set of positive integers.

\subsection{Incomplete information on one side}

Let $K$ be a finite set of parameters and let $S$ and $T$ be two convex sets of actions. For any $k \in K$, let $G^{k}: S \times T \rightarrow \mathbb{R}$ be the payoff function of a normal-form game. Assume that $G^{k}$ is bi-linear for all $k$ and that $\|G\|:=\sup _{k, s, t}\left|G^{k}(s, t)\right|<\infty$.

\subsubsection{The primal game $\mathcal{G}(p)$}

For any $p \in \Delta(K)$, one defines a game of incomplete information on one side, denoted by $\mathcal{G}(p)$, as follows:

- Before the play, $k \in K$ is chosen according to $p$ and told to player 1 .

- Then, the game $G^{k}$ is played, i.e. player 1 (resp. 2) chooses $s \in S$ (resp. $t \in T$ ).

The set of strategies of player 1 (resp. 2) is $S^{K}$ (resp. T). For any $(\hat{s}, t) \in S^{K} \times T$, the payoff is given by

$$
\gamma(p, \hat{s}, t):=\sum_{k \in K} p^{k} G^{k}\left(\hat{s}^{k}, t\right)
$$

Let $v^{-}(p)$ and $v^{+}(p)$ denote, respectively, the maxmin and minmax of $\mathcal{G}(p)$, i.e.

$$
\begin{aligned}
v^{-}(p) & :=\sup _{\hat{s} \in S^{K}} \inf _{t \in T} \gamma(p, \hat{s}, t), \\
v^{+}(p) & :=\inf _{t \in T} \sup _{\hat{s} \in S^{K}} \gamma(p, \hat{s}, t) .
\end{aligned}
$$




\subsubsection{The dual game $\mathcal{D}[\mathcal{G}](x)$}

To each $x \in \mathbb{R}^{K}$, corresponds the dual game $\mathcal{D}[\mathcal{G}](x)$, a modified version of the game where player 1 can choose the parameter $k \in K$ with some cost $x^{k}$. Formally, the set of strategies of player 1 (resp. 2) is $\Delta(K) \times S$ (resp. $T$ ). For any $(p, s)$ and $t$, the payoff is given by

$$
h[x](p, s, t):=\sum_{k \in K} p^{k} G^{k}(s, t)-\langle p, x\rangle .
$$

Let $w^{-}(p)$ and $w^{+}(p)$ denote, respectively, the maxmin and minmax of $\mathcal{D}[\mathcal{G}](x)$, i.e.

$$
\begin{aligned}
w^{-}(p) & :=\sup _{(p, s) \in \Delta(K) \times S} \inf _{t \in T} h[x](p, s, t), \\
w^{+}(p) & :=\inf _{t \in T} \sup _{(p, s) \in \Delta(K) \times S} h[x](p, s, t) .
\end{aligned}
$$

\subsubsection{Duality results}

Let $v^{+}(p), v^{-}(p)$ and $w^{+}(x), w^{-}(x)$ be the minmax and maxmin of $\mathcal{G}(p)$ and $\mathcal{D}[\mathcal{G}](x)$, respectively, for any $p \in \Delta(K)$ and $x \in \mathbb{R}^{K}$. Then, essentially, $v^{+}$and $w^{+}$(resp. $v^{-}$and $w^{-}$) are Fenchel conjugates to each other (see $[4,5]$ ). An important consequence of these relations is that $\varepsilon$-optimal strategies for player 2 in $\mathcal{D}[\mathcal{G}](x)$ are also $\varepsilon$-optimal for him in $\mathcal{G}(p)$, for an appropriate choice of $(p, x)$.

Start by defining two transforms which are closely related to the Fenchel operator, yet more adapted to this framework.

Definition 2.2. Let $f: \mathbb{R}^{K} \rightarrow \mathbb{R}$. Define its upper and lower conjugates $f^{\sharp}, f^{b}: \mathbb{R}^{K} \rightarrow \mathbb{R}$ by:

$$
\begin{aligned}
f^{\sharp}(x) & :=\sup _{y \in \mathbb{R}^{K}} f(y)-\langle y, x\rangle, \\
f^{b}(y) & :=\inf _{x \in \mathbb{R}^{K}} f(x)+\langle x, y\rangle .
\end{aligned}
$$

Recall that the Fenchel conjugate of $f$ is given by $f^{*}(x)=\sup _{y \in \mathbb{R}^{K}}\langle y, x\rangle-f(y)$. Thus,

$$
f^{\sharp}(x)=(-f)^{*}(-x), \quad \text { and } \quad f^{b}(y)=-f^{*}(-y), \quad \forall x, y \in \mathbb{R}^{K} .
$$

Define the superdifferential of $f$ at $x$ as usual:

$$
\partial^{+} f(x):=\left\{y \in \mathbb{R}^{K} \mid f(x)+\left\langle y, x^{\prime}-x\right\rangle \geq f\left(x^{\prime}\right), \forall x^{\prime} \in \mathbb{R}^{K}\right\} .
$$

Notation 2.3. Without further mention, the upper and lower value functions, defined on $\Delta(K)$, are extended to $\mathbb{R}^{K}$ by $-\infty$ in $\mathbb{R}^{K} \backslash \Delta(K)$.

We refer to [11, Chapter 2] for the following results.

Theorem. The map $v^{+}: \Delta(K) \rightarrow \mathbb{R}$ is concave and $\|G\|$-Lipschitz continuous. Moreover, for all $p \in \Delta(K)$ and $x \in \mathbb{R}^{K}$,

$$
\begin{array}{llll}
w^{+}(x)=\left(v^{+}\right)^{\sharp}(x), & \text { and } & v^{+}(p)=\left(w^{+}\right)^{b}(p) . \\
w^{-}(x)=\left(v^{-}\right)^{\sharp}(x), & \text { and } & v^{-}(p)=\left(w^{-}\right)^{b}(p) .
\end{array}
$$

Corollary. Given $p \in \Delta(K)$, let $x \in \partial^{+} v^{+}(p)$, and let $t_{\varepsilon} \in T$ be an $\varepsilon$-optimal strategy of player 2 in $\mathcal{D}[\mathcal{G}](x)$. Then, $t_{\varepsilon}$ is $\varepsilon$-optimal for player 2 in $\mathcal{G}(p)$. 


\subsection{Incomplete information on two sides}

Let $K$ and $L$ be two finite set of parameters and let $S$ and $T$ be two convex sets of actions. For any $(k, \ell) \in K \times L$, let $G^{k \ell}: S \times T \rightarrow \mathbb{R}$ be the payoff function of a normal-form game. Assume that $G^{k \ell}$ is bi-linear for all $(k, \ell)$ and that $\|G\|:=\sup _{k, s, t}\left|G^{k}(s, t)\right|<\infty$.

\subsubsection{The primal game $\mathcal{G}(\pi)$}

For any $\pi \in \Delta(K \times L)$, define a game of incomplete information on two sides, denoted by $\mathcal{G}(\pi)$, as follows:

- Before the play, $(k, \ell) \in K \times L$ is chosen according to $\pi$. Player 1 (resp. 2) is informed of $k$ (resp. $\ell$ ).

- Then, the game $G^{k \ell}$ is played, i.e. Player 1 chooses $s \in S$, player 2 chooses $t \in T$ and the payoff is $G^{k \ell}(s, t)$.

The set of strategies of player 1 (resp. 2) is $S^{K}$ (resp. $\left.T^{L}\right)$. For any $(\hat{s}, \hat{t}) \in S^{K} \times T^{L}$, the payoff is given by

$$
\gamma(\pi, \hat{s}, \hat{t}):=\sum_{(k, \ell) \in K \times L} \pi^{k \ell} G^{k \ell}\left(\hat{s}^{k}, \hat{t}^{\ell}\right) .
$$

Let $v^{-}(\pi)$ (resp. $v^{+}(\pi)$ ) be the maxmin (resp. minmax) of $\mathcal{G}(\pi)$.

Remark 2.4. Incomplete information on one side corresponds to the case $|L|=1$.

In order to apply the duality techniques mentioned above, it is convenient to give an equivalent formulation of the primal game. The idea is to use the duality operator with respect to the first marginal of $\pi$.

\subsubsection{The intermediate game $\mathcal{G}_{Q}(p)$}

Let $Q \in \Delta(L)^{K}$ be a fixed matrix of conditional probabilities, i.e. for all $k \in K, Q(\cdot \mid k)$ is a probability distribution on $L$. For each $k \in K$, define the game $G_{Q}^{k}$ as follows:

- Before the play, $\ell \in L$ is chosen according to $Q(\cdot \mid k) \in \Delta(L)$ and told to player 2 .

- Then, the game $G^{k \ell}$ is played. Player 1 (resp. 2) chooses $s \in S$ (resp. $t \in T$ ) and the payoff is $G^{k \ell}(s, t)$.

The set of strategies of player 1 (resp. 2) is $S$ (resp. $T^{L}$ ). For any $(s, \hat{t}) \in S \times T^{L}$, the payoff is given by

$$
G_{Q}^{k}(s, \hat{t}):=\sum_{\ell \in L} Q(\ell \mid k) G^{k \ell}\left(s, \hat{t}^{\ell}\right)
$$


Note that $G_{Q}^{k}$ is bi-linear for all $k \in K$ and that $\sup _{k, s, \hat{t}}\left|G_{Q}^{k}(s, \hat{t})\right| \leq\|G\|<\infty$. Besides, $T^{L}$ is a convex set. Hence, $\left(S, T^{L},\left\{G_{Q}^{k}, k \in K\right\}\right)$ satisfies the assumptions of Section 2.1. For any $p \in \Delta(K)$, define the game $\mathcal{G}_{Q}(p)$ as in Section 2.1.1.

\subsubsection{Duality results: extension to the general case}

Let $v_{Q}^{+}(p)$ be the minmax of $\mathcal{G}_{Q}(p)$, for any $p \in \Delta(K)$. As in Section 2.1.2 define, for any $x \in \mathbb{R}^{K}$, the dual game $\mathcal{D}\left[\mathcal{G}_{Q}\right](x)$, and let $w_{Q}^{+}(x)$ denote its minmax. Then the following result holds.

Theorem 2.5. For all $Q \in \Delta(L)^{K}$, the maps $v_{Q}^{+}: \Delta(K) \rightarrow \mathbb{R}$ is concave and $\|G\|$-Lipschitz continuous. Moreover, for any $p \in \Delta(K)$ and $x \in \mathbb{R}^{K}$,

$$
\begin{array}{lll}
w_{Q}^{+}(x)=\left(v_{Q}^{+}\right)^{\sharp}(x), & \text { and } & v_{Q}^{+}(p)=\left(w_{Q}^{+}\right)^{b}(p) . \\
w_{Q}^{-}(x)=\left(v_{Q}^{-}\right)^{\sharp}(x), & \text { and } & v_{Q}^{-}(p)=\left(w_{Q}^{-}\right)^{b}(p) .
\end{array}
$$

Note that the games $\mathcal{G}(\pi)$ and $\mathcal{G}_{Q}(p)$ are identical whenever $\pi=p \otimes Q$ (i.e. $\pi^{k \ell}=p^{k} Q(\ell \mid k)$ for all $(k, \ell) \in K \times L)$. Together with the corollary in Section 2.1.3, this observation yields the following result.

Corollary 2.6. Let $\pi \in \Delta(K \times L)$ and let $(p, Q) \in \Delta(K) \times \Delta(L)^{K}$ be such that $\pi=p \otimes Q$. Let $x \in \partial^{+} v_{Q}^{+}(p)$, and let $\hat{t}_{\varepsilon} \in T^{L}$ be an $\varepsilon$-optimal strategy of player 2 in $\mathcal{D}\left[\mathcal{G}_{Q}\right](x)$. Then, $\hat{t}_{\varepsilon}$ is $\varepsilon$-optimal for player 2 in $\mathcal{G}(\pi)$.

Our model being symmetric (i.e. the players play the same role), one defines in the same manner a dual game with respect to the second marginal of $\pi$ and analogue results are obtained. In the sequel, we will only work with the dual game with respect to the first marginal on $\pi$. Thus, we will focus on the construction of optimal strategies for player 2 .

\section{Repeated games with incomplete information}

Let $K, L, I, J$ be finite sets. A repeated game with incomplete information is described by a family of $I \times J$-matrix games $\left\{G^{k \ell},(k, \ell) \in K \times L\right\}$ and a probability $\pi \in \Delta(K \times L)$. A pair of parameters $(k, \ell) \in K \times L$ is drawn according to $\pi \in \Delta(K \times L)$. Player 1 is informed of $k$, player 2 is informed of $\ell$. Then, the game $G^{k \ell}$ is played repeatedly: the players choose actions $\left(i_{m}, j_{m}\right) \in I \times J$ at each stage $m \geq 1$. We assume that the players observe and remember the history of the play, $h_{m}=\left(i_{1}, j_{1}, \ldots, i_{m-1}, j_{m-1}\right)$. The set of possible histories at stage $m$ is $\mathcal{H}_{m}:=(I \times J)^{m-1}$ and $\mathcal{H}:=\cup_{m \geq 1} \mathcal{H}_{m}$ is the set of all possible (finite) histories. The information available to player 1 (resp. 2) at stage $m$ is $\left(k, h_{m}\right)\left(\right.$ resp. $\left.\left(\ell, h_{m}\right)\right)$.

Definition 3.1 (Strategies). A strategy of player 1 (resp. 2) is a function $\hat{s}: K \times \mathcal{H} \rightarrow \Delta(I)$ (resp. $\hat{t}: L \times \mathcal{H} \rightarrow \Delta(J)$ ). 
By a slight abuse in the notation, the set of strategies are denoted, respectively, by $S^{K}$ and $T^{L}$. Let $\mathbb{P}_{\hat{s}, \hat{t}}^{\pi}$ be the unique probability distribution over $K \times L \times(I \times J)^{\infty}$ induced by $\pi \in \Delta(K \times L)$ and $(\hat{s}, \hat{t}) \in S^{K} \times T^{L}$. The payoff function in $\mathcal{G}_{\theta}(\pi)$ is defined as follows:

$$
\forall(\hat{s}, \hat{t}) \in S^{K} \times T^{L}, \quad \gamma_{\theta}(\pi, \hat{s}, \hat{t}):=\mathbb{E}_{\hat{s}, \hat{t}}^{\pi}\left[\sum_{m \geq 1} \theta_{m} G^{k \ell}\left(i_{m}, j_{m}\right)\right],
$$

where $\mathbb{E}_{\hat{s}, \hat{t}}^{\pi}$ is the expectation with respect to $\mathbb{P}_{\hat{s}, \hat{t}}^{\pi}$. The maxmin and minmax of $\Gamma_{\theta}(\pi)$ are, respectively:

$$
\begin{aligned}
& v_{\theta}^{-}(\pi):=\sup _{\hat{s} \in S^{K}} \inf _{\hat{t} \in T^{L}} \gamma_{\theta}(\pi, \hat{s}, \hat{t}), \\
& v_{\theta}^{+}(\pi):=\inf _{\hat{t} \in T^{L}} \sup _{\hat{s} \in S^{K}} \gamma_{\theta}(\pi, \hat{s}, \hat{t}) .
\end{aligned}
$$

The game $\mathcal{G}_{\theta}(\pi)$ has a value if $v_{\theta}^{-}(\pi)=v_{\theta}^{+}(\pi)$.

Lemma 3.2. For any $\pi \in \Delta(K \times L)$, the game $\mathcal{G}_{\theta}(\pi)$ has a value, denoted by $v_{\theta}(\pi)$.

Proof. Fix $\varepsilon>0$. Let $m_{\varepsilon}$ be such that $\sum_{m \geq m_{\varepsilon}} \theta_{m} \leq \varepsilon /\|G\|$. Let $\rho_{\theta, \varepsilon}(\pi)$ be the value of the finite game obtained by truncating the stream of payoffs at stage $m_{\varepsilon}$. Then, clearly,

$$
\left|v_{\theta}^{-}(\pi)-\rho_{\theta, \varepsilon}(\pi)\right| \leq \varepsilon, \quad \text { and } \quad\left|v_{\theta}^{+}(\pi)-\rho_{\theta, \varepsilon}(\pi)\right| \leq \varepsilon, \quad \forall \pi \in \Delta(K \times L) .
$$

Thus $v_{\theta}^{+}(\pi) \leq v_{\theta}^{-}(\pi)+2 \varepsilon$, for all $\pi \in \Delta(K \times L)$.

\subsection{Recursive structure}

At stage 1 , the information of player 1 (resp. 2) is simply $k$ (resp. $\ell$ ). His strategy is denoted by $\sigma:=\hat{s}\left(h_{1}\right) \in \Delta(I)^{K}$ (resp. $\left.\tau:=\hat{t}\left(h_{1}\right) \in \Delta(J)^{L}\right)$.

Definition 3.3. For any $(i, j) \in I \times J$ and $\hat{s} \in S^{K}$ define the continuation strategy $\hat{s}_{i j}^{+} \in S^{K}$ as follows:

$$
\hat{s}_{i j}^{+}: \mathcal{H} \rightarrow \Delta(I), \quad h \mapsto \hat{s}(i, j, h),
$$

where for any $h \in \mathcal{H},(i, j, h)$ denotes the history obtained by concatenating $(i, j)$ and $h$.

Similarly, one defines continuation strategies $\hat{t}_{i j}^{+} \in T^{L}$ for player 2. The weight of the remaining stages after stage 1 is $\sum_{m \geq 2} \theta_{m}=1-\theta_{1}$. If $\theta_{1}<1$, it is convenient to consider the normalized evaluation $\theta^{+} \in \Delta\left(\mathbb{N}^{*}\right)$, defined as:

$$
\theta_{m}^{+}:=\frac{\theta_{m+1}}{1-\theta_{1}}, \quad m \geq 1
$$

Let $\mathbb{P}_{\sigma, \tau}^{\pi}$ denote the unique probability measure on $K \times L \times I \times J$ induced by $(\sigma, \tau) \in$ $\Delta(I)^{K} \times \Delta(J)^{L}$ and $\pi \in \Delta(K \times L)$. Explicitly,

$$
\mathbb{P}_{\sigma, \tau}^{\pi}(k, \ell, i, j)=\pi^{k \ell} \sigma^{k}(i) \tau^{\ell}(j), \quad \forall(k, \ell, i, j) \in K \times L \times I \times J .
$$


Let $\pi_{i j}:=\mathbb{P}_{\sigma, \tau}^{\pi}(\cdot, \cdot \mid i, j) \in \Delta(K \times L)$ be the conditional probability on $K \times L$, given $(i, j) \in I \times J$. It is defined arbitrarily if $\mathbb{P}_{\sigma, \tau}^{\pi}(i, j)=0$. Otherwise, for all $(k, \ell) \in K \times L$,

$$
\pi_{i j}^{k \ell}=\frac{\pi^{k \ell} \sigma^{k}(i) \tau^{\ell}(j)}{\sum_{(k, \ell) \in K \times L} \pi^{k \ell} \sigma^{k}(i) \tau^{\ell}(j)} .
$$

Now, one can easily write a recursive formula for the payoff function, considering the first stage and the continuation game separately:

$$
\gamma_{\theta}(\pi, \hat{s}, \hat{t})=\theta_{1} G_{\sigma \tau}^{\pi}+\left(1-\theta_{1}\right) \sum_{(i, j) \in I \times J} \mathbb{P}_{\sigma \tau}^{\pi}(i, j) \gamma_{\theta^{+}}\left(\pi_{i j}, \hat{s}_{i j}^{+}, \hat{t}_{i j}^{+}\right),
$$

where $G_{\sigma \tau}^{\pi}:=\mathbb{E}_{\sigma, \tau}^{\pi}\left[G^{k \ell}(i, j)\right]$ denotes the expected payoff at stage 1 . By convention we put $\gamma_{\theta^{+}}=0$ if $\theta_{1}=1$.

The following proposition is due to Mertens and Zamir [8, Section 3]. For convenience, we provide here a direct and shorter proof.

Proposition 3.4 (Primal recursive formula). For any $\pi \in \Delta(K \times L)$ and $\theta \in \Delta\left(\mathbb{N}^{*}\right)$ :

$$
\begin{aligned}
v_{\theta}(\pi) & =\sup _{\sigma \in \Delta(I)^{K}} \inf _{\tau \in \Delta(J)^{L}}\left\{\theta_{1} G_{\sigma \tau}^{\pi}+\left(1-\theta_{1}\right) \sum_{(i, j) \in I \times J} \mathbb{P}_{\sigma \tau}^{\pi}(i, j) v_{\theta^{+}}\left(\pi_{i j}\right)\right\} . \\
& =\inf _{\tau \in \Delta(J)^{L}} \sup _{\sigma \in \Delta(I)^{K}}\left\{\theta_{1} G_{\sigma \tau}^{\pi}+\left(1-\theta_{1}\right) \sum_{(i, j) \in I \times J} \mathbb{P}_{\sigma \tau}^{\pi}(i, j) v_{\theta^{+}}\left(\pi_{i j}\right)\right\} .
\end{aligned}
$$

Proof. Consider the maxmin. Let $\hat{s}=\left(\sigma, \hat{s}^{+}\right) \in S^{K}$ be a strategy of player 1. Let $\hat{t}^{+}$be such that, for each $(i, j) \in I \times J, \hat{t}_{i j}^{+}$is a best reply to $\hat{s}_{i j}^{+}$in $\mathcal{G}_{\theta^{+}}\left(\pi_{i j}\right)$. Then, for all $(\sigma, \tau) \in \Delta(I)^{K} \times \Delta(J)^{L}$,

$$
\gamma_{\theta}(\pi, \hat{s}, \hat{t}) \leq \theta_{1} G_{\sigma \tau}^{\pi}+\left(1-\theta_{1}\right) \sum_{(i, j) \in I \times J} \mathbb{P}_{\sigma \tau}^{\pi}(i, j) v_{\theta^{+}}\left(\pi_{i j}\right) .
$$

Player 1 can still maximize over his own first-stage strategy. A best reply of player 2 yields

$$
\begin{aligned}
v_{\theta}(\pi) & =\sup _{\left(\sigma, \hat{s}^{+}\right)} \inf _{\left(\tau, \hat{t}^{+}\right)} \gamma_{\theta}(\pi, \hat{s}, \hat{t}), \\
& \leq \max _{\sigma \in \Delta(I)^{K}} \min _{\tau \in \Delta(J)^{L}}\left\{\theta_{1} G_{\sigma \tau}^{\pi}+\left(1-\theta_{1}\right) \sum_{(i, j) \in I \times J} \mathbb{P}_{\sigma \tau}^{\pi}(i, j) v_{\theta^{+}}\left(\pi_{i j}\right)\right\} .
\end{aligned}
$$

Reversing the roles of the players one obtains, symmetrically:

$$
v_{\theta}(\pi) \geq \min _{\tau \in \Delta(J)^{L}} \max _{\sigma \in \Delta(I)^{K}}\left\{\theta_{1} G_{\sigma \tau}^{\pi}+\left(1-\theta_{1}\right) \sum_{(i, j) \in I \times J} \mathbb{P}_{\sigma \tau}^{\pi}(i, j) v_{\theta^{+}}\left(\pi_{i j}\right)\right\},
$$

and the result follows then because maxmin $\leq$ minmax.

Comments Proposition 3.4 provides a recurrence formula satisfied by the values. However, none of the players can "use it", since the computation of the conditional probability $\pi_{i j}$ involves the strategy of his opponent. The situation contrasts with the case of repeated games with incomplete information on one side (i.e. $L$ is a singleton). In this case, player 1 can compute (and controls) the conditional probabilities, so that this formula provides an explicit, recursive way to construct optimal strategies for him (see [11, Section 3]). 


\subsection{The dual game}

Consider, for any $(x, Q) \in \mathbb{R}^{K} \times \Delta(L)^{K}$, the first dual game $\mathcal{D}\left[\mathcal{G}_{\theta}\right](x, Q)$, i.e. the dual game with respect to the first marginal of $\pi$. The set of strategies of player 1 (resp. 2) is $\Delta(K) \times S^{K}$ (resp. $\left.T^{L}\right)$ and, for any $(p, \hat{s}, \hat{t}) \in \Delta(K) \times S^{K} \times T^{L}$, the payoff is given by

$$
h_{\theta}[x, Q](p, \hat{s}, \hat{t})=\gamma_{\theta}(p \otimes Q, \hat{s}, \hat{t})-\langle p, x\rangle .
$$

The maxmin and minmax are defined, respectively, as:

$$
\begin{aligned}
w_{\theta}^{-}(x, Q) & :=\sup _{(p, \hat{s}) \in \Delta(K) \times S^{K}} \inf _{\hat{t} \in T^{L}} h_{\theta}[x, Q](p, \hat{s}, \hat{t}), \\
w_{\theta}^{+}(x, Q) & :=\inf _{\hat{t} \in T^{L}} \sup _{(p, \hat{s}) \in \Delta(K) \times S^{K}} h_{\theta}[x, Q](p, \hat{s}, \hat{t}) .
\end{aligned}
$$

The existence of the value follows from Lemma 3.2 and Theorem 2.5.

Let $(\sigma, \tau) \in \Delta(I)^{K} \times \Delta(J)^{L}$ be a pair of first-stage strategies. Let $\pi \in \Delta(K \times L), p \in \Delta(K)$ and $Q \in \Delta(L)^{K}$ be such that $\pi=p \otimes Q$. For any $(i, k) \in I \times K$, define

$$
\begin{aligned}
p_{i}^{k} & :=\mathbb{P}_{\sigma, \tau}^{\pi}(k \mid i), \\
G_{i \tau}^{k, Q} & =\mathbb{E}_{\sigma, \tau}^{\pi}\left[G^{k \ell}(i, j) \mid k, i\right] .
\end{aligned}
$$

The corresponding vectors are denoted by $p_{i} \in \Delta(K)$ and $G_{i \tau}^{Q} \in \mathbb{R}^{K}$, respectively. The former is the posterior probability on $K$ given $i$, the latter is the vector of expected payoffs for player 2, after he observes the action played by his opponent. Explicitly,

$$
G_{i \tau}^{k, Q}=\sum_{(\ell, j) \in L \times J} Q(\ell \mid k) \tau^{\ell}(j) G^{k \ell}(i, j)
$$

As in (3.1), the following recurrence formula for the payoff function is straightforward:

$$
h_{\theta}[x, Q](p, \hat{s}, \hat{t})=\left(1-\theta_{1}\right) \sum_{i \in I} \mathbb{P}_{\sigma, \tau}^{\pi}(i)\left(\sum_{j \in J} \mathbb{P}_{\sigma, \tau}^{\pi}(j \mid i) \gamma_{\theta^{+}}\left(\pi_{i j}, \hat{s}_{i j}^{+}, \hat{t}_{i j}^{+}\right)-\left\langle p_{i}, \frac{x-\theta_{1} G_{i \tau}^{Q}}{1-\theta_{1}}\right\rangle\right) .
$$

\section{The conditional probabilities}

Let us give here a closer look at the conditional probability $\pi_{i j}=\mathbb{P}_{\sigma, \tau}^{\pi}(\cdot, \cdot \mid i, j) \in \Delta(K \times L)$. The next lemma provides a decomposition which will play a key role in the sequel.

Lemma 3.5. For any $(i, j)$ such that $\mathbb{P}_{\sigma, \tau}^{\pi}(i, j)>0$, one can express $\pi_{i j}$ as $p_{i j} \otimes Q_{j}$, where $p_{i j} \in \Delta(K)$ and $Q_{j} \in \Delta(L)^{K}$ are defined as follows:

$$
\begin{aligned}
p_{i j}^{k} & =p_{i}^{k} \frac{\mathbb{P}_{\sigma, \tau}^{\pi}(j \mid k)}{\mathbb{P}_{\sigma, \tau}^{\pi}(j \mid i)}, \quad \forall k \in K \\
Q_{j}(\ell \mid k) & =\frac{Q(\ell \mid k) \tau^{\ell}(j)}{\sum_{\ell^{\prime}} Q\left(\ell^{\prime} \mid k\right) \tau^{\ell^{\prime}}(j)}, \quad \forall(k, \ell) \in K \times L .
\end{aligned}
$$


Proof. Fix $(i, j) \in I \times J$ such that $\mathbb{P}_{\sigma, \tau}^{\pi}(i, j)>0$, and $(k, \ell) \in K \times L$. By disintegration, one has:

$$
\pi_{i j}^{k \ell}=\mathbb{P}_{\sigma, \tau}^{\pi}(k \mid i, j) \mathbb{P}_{\sigma, \tau}^{\pi}(\ell \mid k, i, j)
$$

where $\mathbb{P}_{\sigma, \tau}^{\pi}(k \mid i, j)$ satisfies

$$
\mathbb{P}_{\sigma, \tau}^{\pi}(k \mid i, j) \mathbb{P}_{\sigma, \tau}^{\pi}(j \mid i)=\mathbb{P}_{\sigma, \tau}^{\pi}(k \mid i) \mathbb{P}_{\sigma, \tau}^{\pi}(j \mid k, i) .
$$

Then (3.6) is obtained from (3.3) and from computing

$$
\mathbb{P}_{\sigma, \tau}^{\pi}(j \mid k, i)=\frac{p^{k} \sigma^{k}(i) \sum_{\ell \in L} Q(\ell \mid k) \tau^{\ell}(j)}{p^{k} \sigma^{k}(i)}=\mathbb{P}_{\sigma, \tau}^{\pi}(j \mid k) .
$$

On the other hand, a direct computation yields

$$
\mathbb{P}_{\sigma, \tau}^{\pi}(\ell \mid k, i, j)=\frac{p^{k} \sigma^{k}(i) Q(\ell \mid k) \tau^{\ell}(j)}{p^{k} \sigma^{k}(i) \sum_{\ell^{\prime} \in L} Q\left(\ell^{\prime} \mid k\right) \tau^{\ell^{\prime}}(j)}=Q_{j}(\ell \mid k) .
$$

\section{Comments}

- An important consequence of Lemma 3.5 is the fact that player 2 can compute (and controls) the matrix of conditional probabilities $Q_{j} \in \Delta(L)^{K}$, for all $j \in J$. Note also that, for all $k \in K$, player 2 can compute

$$
\mathbb{P}_{\sigma, \tau}^{\pi}(j \mid k)=\sum_{\ell \in L} Q(\ell \mid k) \tau^{\ell}(j)
$$

The vector $\left(\mathbb{P}_{\sigma, \tau}^{\pi}(j \mid k)\right)_{k \in K}$ appears in the construction of optimal strategies for player 2 (see Proposition 3.7), allowing to adjust the payoff function in the continuation game.

- In the independent case (i.e. $\pi=p \otimes q$, for some $p \in \Delta(K)$ and $q \in \Delta(L)$ ) one simply has $\pi_{i j}=p_{i} \otimes q_{j}$, where for each $\ell \in L, q_{j}^{\ell}=\frac{q^{\ell} \tau^{\ell}(j)}{\sum_{\ell^{\prime} \in L} q^{\ell^{\prime}} \tau^{\ell^{\prime}}(j)}\left(p_{i}\right.$ is defined similarly). As a consequence, each player can compute and controls one of the marginals of $\pi_{i j}$, and the payoffs need not be adjusted (see Section 3.5 for more details).

\subsection{Main result}

Here, we provide a recursive formula for the values of the dual game, by introducing an auxiliary parameter $\zeta \in \mathbb{R}^{K}$ to the game. For each $k \in K, \zeta^{k}$ keeps track of some adjustments in the payoff function, which allow to decompose the conditional probability distribution $\pi_{i j}$ as $p_{i} \otimes Q_{j}$. The importance of such "separation of variables" will be clear in the proof of Theorem (3.7). Surprisingly, these adjustments are specific to the general case (see Section 3.8 for the independent case). 


\subsubsection{The auxiliary game $\mathcal{G}_{\theta}(\pi ; \zeta)$}

For any $\zeta \in \mathbb{R}^{K}$, let $\mathcal{G}_{\theta}(\pi ; \zeta)$ be the same game as $\mathcal{G}_{\theta}(\pi)$, but with a slightly different payoff function, given by

$$
\gamma_{\theta}(\pi, \hat{s}, \hat{t} ; \zeta)=\mathbb{E}_{\hat{s}, \hat{t}}^{\pi}\left[\sum_{m \geq 1} \theta_{m} \zeta^{k} G^{k \ell}\left(i_{m}, j_{m}\right)\right] .
$$

Note that $\mathcal{G}_{\theta}(\pi ; \mathbf{1})=\mathcal{G}_{\theta}(\pi)$, where $\mathbf{1}$ is the vector of 1 's in $\mathbb{R}^{K}$.

The next result is quite technical, yet important. It states that, after an adequate adjustment of the payoff function, the conditional probability $\pi_{i j}$ can be expressed as $p_{i} \otimes Q_{j}$.

Lemma 3.6. Let $\pi \in \Delta(K \times L)$. Let $(\sigma, \tau) \in \Delta(I)^{K} \times \Delta(J)^{L}$ be a pair of first-stage strategies and let $(i, j) \in I \times J$ be a pair of actions such that $\mathbb{P}_{\sigma, \tau}^{\pi}(i, j)>0$. Then, for any pair of strategies $(\hat{s}, \hat{t}) \in S^{K} \times T^{L}$ and any evaluation $\theta \in \Delta\left(\mathbb{N}^{*}\right)$ :

$$
\mathbb{P}_{\sigma, \tau}^{\pi}(j \mid i) \gamma_{\theta}\left(\pi_{i j}, \hat{s}, \hat{t} ; \zeta\right)=\gamma_{\theta}\left(p_{i} \otimes Q_{j}, \hat{s}, \hat{t} ; \zeta_{j}\right)
$$

where for all $k \in K, \zeta_{j}^{k}:=\zeta^{k} \mathbb{P}_{\sigma, \tau}^{\pi}(j \mid k)$.

Proof. The result follows from Lemma 3.5 by a direct computation. Indeed, for any such $(i, j) \in$ $I \times J$ one has:

$$
\begin{aligned}
& \mathbb{P}_{\sigma, \tau}^{\pi}(j \mid i) \gamma_{\theta}\left(\pi_{i j}, \hat{s}, \hat{t} ; \zeta\right)=\mathbb{P}_{\sigma, \tau}^{\pi}(j \mid i) \sum_{(k, \ell) \in K \times L} \pi_{i j}^{k \ell} \mathbb{E}_{\hat{s}^{k}, \hat{t}^{\ell}}\left[\sum_{m \geq 1} \theta_{m} \zeta^{k} G^{k \ell}\left(i_{m}, j_{m}\right)\right], \\
& =\mathbb{P}_{\sigma, \tau}^{\pi}(j \mid i) \sum_{(k, \ell) \in K \times L} p_{i}^{k} \frac{\mathbb{P}_{\sigma, \tau}^{\pi}(j \mid k)}{\mathbb{P}_{\sigma, \tau}^{\pi}(j \mid i)} Q_{j}(\ell \mid k) \mathbb{E}_{\hat{s}^{k}, \hat{t}^{\ell}}\left[\sum_{m \geq 1} \theta_{m} \zeta^{k} G^{k \ell}\left(i_{m}, j_{m}\right)\right], \\
& =\sum_{(k, \ell) \in K \times L} p_{i}^{k} Q_{j}(\ell \mid k) \mathbb{E}_{\hat{s}^{k}, \hat{t}^{\ell}}\left[\sum_{m \geq 1} \theta_{m}^{+} \mathbb{P}_{\sigma, \tau}^{\pi}(j \mid k) \zeta^{k} G^{k \ell}\left(i_{m}, j_{m}\right)\right], \\
& =\gamma_{\theta}\left(p_{i} \otimes Q_{j}, \hat{s}, \hat{t} ; \zeta_{j}\right) .
\end{aligned}
$$

We are now ready to state our main result, which is the following recursive formula for the values of the auxiliary dual game $\mathcal{D}\left[\mathcal{G}_{\theta}\right](x, Q ; \zeta)$. Its importance relies on the fact that a) it allows player 2 to construct optimal strategies recursively in the dual game, and b) these strategies are optimal in the primal game $\mathcal{G}_{\theta}(\pi)$, due to the duality results in Section 2.2.3, for an appropriate choice of the parameters. The key point here is that player 2 can compute $\left(x_{i j}, Q_{j} ; \zeta_{j}\right)$ and $\theta^{+}$. This is of capital importance in order to construct an optimal strategy for this player (see Section 3.4).

Theorem 3.7 (Dual recursive formula). For all $(x, Q, \zeta, \theta) \in \mathbb{R}^{K} \times \Delta(L)^{K} \times \mathbb{R}^{K} \times \Delta\left(\mathbb{N}^{*}\right)$,

$$
\begin{gathered}
w_{\theta}(x, Q ; \zeta)=\left(1-\theta_{1}\right) \min _{\tau \in \Delta(J)^{L}} \min _{\substack{\left(x_{i j}\right)_{i j} \in\left(\mathbb{R}^{K}\right)^{I \times J} \\
\forall i, \sum_{j} x_{i j}=\frac{x-\theta_{1} G_{i \tau}^{Q}}{1-\theta_{1}}}} \max _{i \in I} \sum_{j \in J} w_{\theta^{+}}\left(x_{i j}, Q_{j} ; \zeta_{j}\right), \\
\end{gathered}
$$

where, for all $(k, j) \in K \times J, \zeta_{j}^{k}:=\zeta^{k} \mathbb{P}_{\sigma, \tau}^{\pi}(j \mid k)$. 
Proof. We divide the proof in two parts, one for each inequality. Strategies $\hat{s} \in S^{K}$ and $\hat{t} \in T^{L}$ are decomposed as $\hat{s}=\left(\sigma, \hat{s}^{+}\right)$and $\hat{t}=\left(\tau, \hat{t}^{+}\right)$with no further mention.

Part 1: The $\geq$ inequality.

Knowing $(p, \sigma) \in \Delta(K) \times \Delta(I)^{K}$ and $\tau \in \Delta(J)^{L}$, player 1 can play, for each $(i, j) \in I \times J$, a best reply to $\hat{t}_{i j}^{+}$in game $\mathcal{G}_{\theta^{+}}\left(\pi_{i j} ; \zeta_{j}\right)$. Then, using the recursive formula for the payoff function, given in (3.5):

$$
h_{\theta}[x, Q](p, \hat{s}, \hat{t}) \geq\left(1-\theta_{1}\right) \sum_{i \in I} \mathbb{P}_{\sigma, \tau}^{\pi}(i)\left(\sum_{j \in J} \mathbb{P}_{\sigma, \tau}^{\pi}(j \mid i) v_{\theta^{+}}\left(\pi_{i j} ; \zeta\right)-\left\langle p_{i}, \frac{x-\theta_{1} G_{i \tau}^{Q}}{1-\theta_{1}}\right\rangle\right) .
$$

Lemma 3.6 implies then that, for any $(i, j) \in I \times J$ such that $\mathbb{P}_{\sigma, \tau}^{\pi}(i, j)>0$,

$$
\mathbb{P}_{\sigma, \tau}^{\pi}(j \mid i) v_{\theta^{+}}\left(\pi_{i j} ; \zeta\right)=v_{\theta^{+}}\left(p_{i} \otimes Q_{j} ; \zeta_{j}\right) .
$$

Clearly, $\mathbb{P}_{\sigma, \tau}^{\pi}(i, k)=p^{k} \sigma^{k}(i)$ is independent of player 2's strategy. Disintegration gives $p \otimes \sigma=$ $\alpha \otimes \mu \in \Delta(K \times I)$, where $\alpha:=(p \otimes \sigma)^{I} \in \Delta(I)$ and $\mu:=(p \otimes \sigma)^{K \mid I} \in \Delta(K)^{I}$. In fact, $\mu(\cdot \mid i)=p_{i}$, for all $i \in I$ occurring with positive probability. Define

$$
p^{\prime} \in \Delta(K) \mapsto f_{j}\left(p^{\prime}\right):=v_{\theta^{+}}\left(p^{\prime} \otimes Q_{j} ; \zeta_{j}\right) .
$$

Let $F:=\sum_{j \in J} f_{j}$. Taking the supremum in $\Delta(K \times I)$ in (3.9) or, equivalently, in $(\alpha, \mu) \in$ $\Delta(I) \times \Delta(K)^{I}$, yields, for all $\hat{t}=\left(\tau, \hat{t}^{+}\right)$:

$$
\begin{aligned}
\sup _{(p, \sigma)} h_{\theta}[x, Q](p, \hat{s}, \hat{t}) & \geq\left(1-\theta_{1}\right) \sup _{\alpha \in \Delta(I)} \sum_{i \in I} \alpha^{i}\left(\sup _{p_{i} \in \Delta(K)} \sum_{j \in J} f_{j}\left(p_{i}\right)-\left\langle p_{i}, \frac{x-\theta_{1} G_{i \tau}^{Q}}{1-\theta_{1}}\right\rangle\right), \\
& =\left(1-\theta_{1}\right) \sup _{i \in I}\left(\sup _{p_{i} \in \Delta(K)} \sum_{j \in J} f_{j}\left(p_{i}\right)-\left\langle p_{i}, \frac{x-\theta_{1} G_{i \tau}^{Q}}{1-\theta_{1}}\right\rangle\right), \\
& =\left(1-\theta_{1}\right) \sup _{i \in I} F^{\sharp}\left(\frac{x-\theta_{1} G_{i \tau}^{Q}}{1-\theta_{1}}\right), \\
& =\left(1-\theta_{1}\right) \sup _{i \in I} \underset{\left(x_{j}\right)_{j} \in\left(\mathbb{R}^{K}\right)^{J}}{\inf } \sum_{j \in J} f_{j}^{\sharp}\left(x_{j}\right) .
\end{aligned}
$$

The last equality is a obtained using a result on the Fenchel conjugates [10, Theorem 16.4]. It relies on the concavity and properness of the functions $f_{j}$, for all $j \in J$. Moreover, the infimum is reached. The proof is left to the Appendix, at the end of this chapter. On the other hand, for all $j \in J$,

$$
\begin{aligned}
f_{j}^{\sharp}\left(x_{j}\right) & =\sup _{p_{i} \in \Delta(K)} v_{\theta^{+}}\left(p_{i} \otimes Q_{j} ; \zeta_{j}\right)-\left\langle p_{i}, x_{j}\right\rangle, & \text { (by def.) } \\
& =w_{\theta^{+}}\left(x_{j}, Q_{j} ; \zeta_{j}\right), & \text { (by Theorem 2.5). }
\end{aligned}
$$

The $\geq$ inequality in (3.8) follows by taking the infimum in $\hat{t}=\left(\tau, \hat{t}^{+}\right)$. To obtain (3.8), commute the supremum and the infimum using that $\sup _{a \in A} \inf _{b \in B}=\inf _{(b(a))_{a} \in B^{A}} \sup _{a \in A}$. We already 
noticed that the infima are reached, the supremum is reached by finiteness.

Part 2: The $\leq$ inequality.

Let $\tau \in \Delta(J)^{\bar{L}}$ and $\left(x_{i j}\right)_{j} \in\left(\mathbb{R}^{K}\right)^{J}$ be optimal in (3.8), for each $i \in I$. The infimum in $\tau$ is reached by compactness and continuity. Let $\hat{t}_{i j}^{+}$be optimal in $\mathcal{D}\left[\mathcal{G}_{\theta^{+}}\right]\left(x_{i j}, Q_{j} ; \zeta_{j}\right)$ for each $(i, j) \in I \times J$. Then, by (3.5), and by the choice of $\left(x_{i j}\right)_{j}$ one has, for any strategy $\left(p, \sigma, \hat{s}^{+}\right)=\left(\alpha, \mu, \hat{s}^{+}\right)$of player 1 ,

$$
\begin{aligned}
h_{\theta}[x, Q](p, \hat{s}, \hat{t}) & =\left(1-\theta_{1}\right) \sum_{i \in I} \alpha^{i} \sum_{j \in J} \gamma_{\theta^{+}}\left(p_{i} \otimes Q_{j}, \hat{s}_{i j}^{+}, \hat{t}_{i j}^{+} ; \zeta_{j}\right)-\left\langle p_{i}, \frac{x-\theta_{1} G_{i \tau}^{Q}}{1-\theta_{1}}\right\rangle, \\
& \leq\left(1-\theta_{1}\right) \sup _{i \in I} \sum_{j \in J}\left(\gamma_{\theta^{+}}\left(p_{i} \otimes Q_{j}, \hat{s}_{i j}^{+}, \hat{t}_{i j}^{+} ; \zeta_{j}\right)-\left\langle p_{i}, x_{i j}\right\rangle\right), \\
& \leq\left(1-\theta_{1}\right) \sup _{i \in I} \sum_{j \in J} w_{\theta^{+}}\left(x_{i j}, Q_{j} ; \zeta_{j}\right), \\
& \leq\left(1-\theta_{1}\right) \inf _{\tau \in \Delta(J)^{L}} \sup _{i \in I} \inf _{\substack{\left(x_{j}\right)_{j} \in\left(\mathbb{R}^{K}\right)^{J} \\
\sum_{j} x_{j}=\frac{x-\theta_{1} G_{i \tau}^{Q}}{1-\theta_{1}}}} \sum_{j \in J} w_{\theta^{+}}\left(x_{j}, Q_{j} ; \zeta_{j}\right) .
\end{aligned}
$$

Thus, player 2 can ensure that the payoff in $\mathcal{D}\left[\mathcal{G}_{\theta}\right](x, Q, \zeta)$ is smaller than the right-hand-side of the last equation. The desired inequality follows.

\section{Comments}

- The recurrence formula implies, in particular, the following result. For any $(x, Q, \zeta, \theta) \in$ $\mathbb{R}^{K} \times \Delta(L)^{K} \times \mathbb{R}^{K} \times \Delta\left(\mathbb{N}^{*}\right)$,

$$
w_{\theta}(x, Q) \leq\left(1-\theta_{1}\right) \min _{\bar{\tau} \in \Delta(J)} \max _{i \in I} w_{\theta^{+}}\left(\frac{x-\theta_{1} G_{i \bar{\tau}}^{Q}}{1-\theta_{1}}, Q\right) .
$$

Let $\left(\tau,\left(x_{i j}\right)_{i j}\right)$ be a minimizer in recurrence formula (3.8). Suppose that player 2's strategy is non-revealing, i.e. that there exists $\bar{\tau} \in \Delta(J)$ such that $\tau^{\ell}(j)=\bar{\tau}(j)$, for all $\ell \in L$. Then, the convexity of $w_{\theta^{+}}(\cdot, Q)$ yields:

$$
w_{\theta}(x, Q)=\left(1-\theta_{1}\right) \max _{i \in I} w_{\theta^{+}}\left(\frac{x-\theta_{1} G_{i \tau}^{Q}}{1-\theta_{1}}, Q\right) .
$$

Conversely, if the equality holds in (3.11), then it is optimal for player 2 to play nonrevealing. It is enough to choose, in the recurrence formula, $\left(\tau,\left(x_{i j}\right)_{i j}\right)$ such that $\tau^{\ell}(j)=$ $\bar{\tau}(j)$, for all $\ell \in L$, for some $\bar{\tau} \in \Delta(J)$, and $x_{i j}=\frac{x-\theta_{1} G_{i, \tau}^{Q}}{1-\theta_{1}}$, for all $(i, j) \in I \times J$.

These particular cases were already pointed out by Cardaliaguet [2] and Cardaliaguet, Laraki and Sorin [3] in the framework of continuous-time games with incomplete information, in the independent case.

- The extra parameter $\zeta$ played a crucial role in the first part of the proof of Theorem 3.7. The expression (3.10) allowed us to take the upper conjugate of the value function of the continuation game, and thus obtain a lower bound on the payoff that player 1 can defend. The introduction of an additional parameter is not necessary in the independent case, due to the relation $\pi_{i j}=p_{i} \otimes q_{j}$. We refer to [6] or Section 3.5 for more details. 


\subsection{Markovian optimal strategies}

In this section, we deduce from Theorem 3.7 and Corollary 2.6 the construction of optimal strategies for player 2 in the game $\mathcal{G}_{\theta}(\pi)$. These strategies are Markovian, in the sense that they depend on the past history only through the present (of the appropriate variables).

Let $(p, Q) \in \Delta(K) \times \Delta(L)^{K}$ be such that $\pi=p \otimes Q$. Define the mapping $v_{\theta}(\cdot, Q): \Delta(K) \rightarrow \mathbb{R}$ by setting $v_{\theta}\left(p^{\prime}, Q\right):=v_{\theta}\left(p^{\prime} \otimes Q\right)$ for all $p^{\prime} \in \Delta(K)$. Set $\zeta:=\mathbf{1} \in \mathbb{R}^{K}$ and choose some $x$ in $\partial^{+} v_{\theta}(p, Q)$.

We are now ready to describe, recursively, an optimal strategy for player 2 in $\mathcal{G}_{\theta}(\pi)$.

- Let $\tau \in \Delta(J)^{L}$ and $\left(x_{i j}\right)_{(i, j) \in I \times J}$ be optimal in $(3.8)$ at $(x, Q, \zeta, \theta)$.

- Choose $j \in J$ with probability $\tau^{\ell}(j)$, where $\ell$ is player 2's private type.

- Play optimally in $\mathcal{D}\left[\mathcal{G}_{\theta^{+}}\right]\left(x_{i j}, Q_{j} ; \zeta_{j}\right)$, where $i \in I$ is player 1 's action at the first stage.

This strategy is optimal in $\mathcal{D}\left[\mathcal{G}_{\theta}\right](x, Q ; \mathbf{1})=\mathcal{D}\left[\mathcal{G}_{\theta}\right](x, Q)$, thanks to Theorem 3.7. By Corollary 2.6 and the choice of $(x, Q)$, the strategy is also optimal in $\mathcal{G}_{\theta}(\pi)$. At stage $m$, the strategy depends only on the variables $\left(x_{m}, Q_{m}, \zeta_{m}\right)$, constructed recursively, and on the normalized evaluation of the payoff. Explicitly, given the couple of actions $(i, j):=\left(i_{m}, j_{m}\right)$ at stage $m$, $\left(x_{m+1}, Q_{m+1}, \zeta_{m+1}\right):=\left(\left(x_{m}\right)_{i j},\left(Q_{m}\right)_{j},\left(\zeta_{m}\right)_{j}\right)$.

Alternatively, given his own actions $\left(j_{1}, \ldots, j_{m}\right)$, player 2 can use the following explicit expression:

$$
\zeta_{m}^{k}=\sum_{\ell \in L} Q(\ell \mid k) \prod_{n=1}^{m} \tau_{n}^{\ell}\left(j_{n}\right), \quad \forall k \in K, \forall m \geq 1,
$$

where $\tau_{n} \in \Delta(J)^{L}$ is his strategy at stage $n$. Note also that, for any $(k, \ell) \in K \times L$,

$$
Q_{m}(\ell \mid k)=\mathbb{P}_{\hat{s}, \hat{t}}^{\pi}\left(\ell \mid k, h_{m}\right) .
$$

\subsection{The independent case}

The aim of this section is to deduce, from Theorem 3.7 the recursive formula for the independent case established by De Meyer and Marino [6]. In particular, we show that the auxiliary variable is not necessary. Let $\pi=p \otimes q$ be a product measure, for some $p \in \Delta(K)$ and $q \in \Delta(L)$. This is equivalent to saying that the private parameters $k$ and $\ell$ are chosen independently. Let $\mathcal{G}_{\theta}(p, q)$ and $\mathcal{G}_{\theta}(p, q ; \zeta)$ stand, respectively, for $\mathcal{G}_{\theta}(\pi)$ and $\mathcal{G}_{\theta}(\pi ; \zeta)$ in this case. Let $\mathbb{P}_{\sigma, \tau}^{p, q}$ denote the unique probability in $K \times L \times I \times J$ induced by $(\sigma, \tau, p, q)$.

Corollary 3.8. For any $(\theta, x, q) \in \Delta\left(\mathbb{N}^{*}\right) \times \mathbb{R}^{K} \times \Delta(L)$ :

$$
w_{\theta}(x, q)=\left(1-\theta_{1}\right) \min _{\tau \in \Delta(J)^{L}} \min _{\substack{\left(x_{i j}\right)_{i j} \in\left(\mathbb{R}^{K}\right)^{I \times J} \\ \forall i, \sum_{j} \bar{\tau}(j) x_{i j}=\frac{x-\theta_{1} G_{i \tau}^{q}}{1-\theta_{1}}}} \max _{i \in I} \sum_{j \in J} \bar{\tau}(j) w_{\theta^{+}}\left(x_{i j}, q_{j}\right),
$$

where $\bar{\tau}$ is the marginal of $q \otimes \tau$ on $\Delta(J), q_{j}$ is its conditional on $L$ given $j$ and $G_{i \tau}^{q}$ is the vector of expected payoffs, given $k \in K$. 
Proof. By Proposition 3.7, for any $\zeta \in \mathbb{R}^{K}$

$$
w_{\theta}(x, q ; \zeta)=\left(1-\theta_{1}\right) \min _{\tau \in \Delta(J)^{L}} \min _{\substack{\left(x_{i j}\right)_{i j} \in\left(\mathbb{R}^{K}\right)^{I \times J} \\ \forall i, \sum_{j} x_{j}=\frac{x-\theta_{1} G_{i \tau}^{q}}{1-\theta_{1}}}} \max _{i \in I} \sum_{j \in J} w_{\theta^{+}}\left(x_{i j}, q_{j} ; \zeta_{j}\right),
$$

where $\zeta_{j}^{k}=\mathbb{P}_{\sigma, \tau}^{p, q}(j \mid k) \zeta^{k}$, for all $(j, k) \in J \times K$. Clearly, $\mathbb{P}_{\sigma, \tau}^{p, q}(j \mid k)=\sum_{\ell \in L} q^{\ell} \tau^{\ell}(j)=\bar{\tau}(j)$, so that $\zeta_{j}=\bar{\tau}(j) \zeta$, for all $j \in J$. Therefore, for all $j$,

$$
v_{\theta}\left(p, q ; \zeta_{j}\right)=\bar{\tau}(j) v_{\theta}(p, q ; \zeta)
$$

The following property of the conjugate is straightforward:

$$
\forall f: \Delta(K) \rightarrow \mathbb{R}, \forall \alpha \in \mathbb{R} \backslash\{0\}, \forall x \in \mathbb{R}^{K}, \quad(\alpha f)^{\sharp}(x)=\alpha f^{\sharp}\left(\frac{x}{\alpha}\right),
$$

It follows from (3.13) and (3.14) that, for any $j \in J$ such that $\bar{\tau}(j)>0$, one has

$$
w_{\theta^{+}}\left(x_{i j}, q_{j}, \zeta_{j}\right)=\bar{\tau}(j) w_{\theta^{+}}\left(\frac{x_{i j}}{\bar{\tau}(j)}, q_{j} ; \zeta\right) .
$$

The result follows then by a simple change of variables, $y_{i j}:=\frac{x_{i j}}{\bar{\tau}(j)}$, and taking $\zeta=\mathbf{1}$.

\section{Extensions, open problems}

\subsection{Infinite set of actions}

The recursive formula satisfied by the values of the dual game was proved by De Meyer and Marino [6] in the more general framework of infinite actions spaces $I, J$, finite sets $K, L$ and a bounded, measurable payoff function - in the independent case. In order to avoid measurability issues, we did not address this problem in the present work. However, we think that such an extension is possible for general initial probabilities assuming, for example, that $I, J$ are Polish spaces equipped with their Borel $\sigma$-algebras. In order to adapt our proof to this more general framework, one probably needs to prove the existence of measurable selections of $\varepsilon$-best replies and then use Theorem 5.3 in [6] to extend Theorem 6.1 (in the Appendix) to integrals instead of finite sums.

\subsection{Optimal strategies in differential games with incomplete information}

Differential games with incomplete information were introduced by Cardaliaguet [2]. As in repeated games with incomplete information, before the game starts, a pair of parameters $(k, \ell)$ is drawn according to some commonly known probability distribution $\pi$ on $K \times L$. Player 1 is informed of $k$ and player 2 of $\ell$. Then, a differential game is played in which the dynamic and the payoff function depend on both types: each player is thus partially informed about the differential game that is played. The existence and characterisation of the value function was established by Cardaliaguet [2] in the independent case, and extended to the general case by the second author [9]. The proof relies on the geometry of the value function (I-concavity and 
II-convexity) and on a sub-dynamic programming principle satisfied by its Fenchel conjugate (i.e. the value of the dual game), in the spirit of (3.11).

The construction of optimal strategies remains, however, an open problem. Equivalently, one may seek to establish a dynamic programming principle satisfied by the value function of the dual game, as in Theorem 2.5.

\section{Acknowledgements}

The authors are indebted to Sylvain Sorin and Bernard De Meyer for their insight and suggestions. This work is part of the second author's Ph.D. thesis under the supervision of the former. This work was supported by the Swiss National Science Foundation grant 200020-149871/1. The two authors gratefully acknowledge the support by the Agence Nationale de la Recherche, under grant ANR JEUDY, ANR-10-BLAN 0112. The first author gratefully acknowledges the support by the Agence Nationale de la Recherche, under grant ANR GAGA: ANR-13-JS01-0004-01.

\section{Appendix}

\section{A result from convex analysis}

Theorem 6.1 ([10], Theorem 16.4). For any $j \in J$, let $f_{j}$ be a concave, bounded, real function on $\Delta(K)$. Let $F:=\sum_{j} f_{j}$. Then, for any $z \in \mathbb{R}^{K}$ :

$$
F^{\sharp}(z)=\min _{\substack{\left(x_{j}\right)_{j} \in\left(\mathbb{R}^{K}\right)^{J} \\ \sum_{j} x_{j}=z}} \sum_{j \in J} f_{j}^{\sharp}\left(x_{j}\right) .
$$

Proof. For any $\left(\mu, x_{j}\right) \in \Delta(K) \times \mathbb{R}^{K}$, the inequality $f_{j}(\mu)-\left\langle\mu, x_{j}\right\rangle \leq f_{j}^{\sharp}\left(x_{j}\right)$ holds. Summation on $j \in J$ yields that, for any $\left(x_{j}\right)_{j} \in\left(\mathbb{R}^{K}\right)^{J}$ such that $\sum_{j} x_{j}=z$,

$$
F(\mu)-\langle\mu, z\rangle \leq \sum_{j \in J} f_{j}^{\sharp}\left(x_{j}\right) .
$$

Taking the supremum in $\mu$ (on the left-hand side), and the infimum in $\left(x_{j}\right)_{j} \in\left(\mathbb{R}^{K}\right)^{J}$ such that $\sum_{j} x_{j}=z$ (on the right-hand-side) one has:

$$
F^{\sharp}(z) \leq \inf _{\substack{\left(x_{j}\right)_{j} \in\left(\mathbb{R}^{K}\right)^{J} \\ \sum_{j} x_{j}=z}} \sum_{j \in J} f_{j}^{\sharp}\left(x_{j}\right) .
$$

Conversely, let $\mu^{\sharp} \in \Delta(K)$ be such that $F^{\sharp}(z)=\sum_{j \in J} f_{j}\left(\mu^{\sharp}\right)-\left\langle\mu^{\sharp}, z\right\rangle$. Then, $z \in \partial^{+} F\left(\mu^{\sharp}\right)$. The boundedness of the $f_{j}$ 's implies [10, Theorem 23.8] that $\partial^{+} F=\sum_{j \in J} \partial^{+} f_{j}$. Let $\left(x_{j}^{\sharp}\right)_{j \in J} \in\left(\mathbb{R}^{K}\right)^{J}$ be such that $\sum_{j \in J} x_{j}^{\sharp}=z$ and $x_{j}^{\sharp} \in \partial^{+} f_{j}\left(\mu^{\sharp}\right)$, for all $j \in J$. Then,

$$
f_{j}\left(\mu^{\sharp}\right)-\left\langle\mu^{\sharp}, x_{j}^{\sharp}\right\rangle=f_{j}^{\sharp}\left(x_{j}^{\sharp}\right), \quad \forall j \in J .
$$


Summation yields then:

$$
F^{\sharp}(z)=\sum_{j \in J} f_{j}\left(\mu^{\sharp}\right)-\left\langle\mu^{\sharp}, z\right\rangle=\sum_{j \in J} f_{j}^{\sharp}\left(x_{j}^{\sharp}\right) .
$$

\section{References}

[1] R.J. Aumann and M. Maschler, Repeated games with incomplete information, with the collaboration of R. Stearns, MIT Press, 1995.

[2] P. Cardaliaguet, Differential games with asymmetric information, SIAM journal on Control and Optimization 46 (2007), 816-838.

[3] P. Cardaliaguet, R. Laraki, and S. Sorin, A continuous time approach for the asymptotic value in two-person zero-sum repeated games, SIAM Journal on Control and Optimization 50 (2012), 1573-1596.

[4] B. De Meyer, Repeated games and partial differential equations, Mathematics of Operations Research 21 (1996), 209-236.

[5] B. De Meyer, Repeated games, duality and the Central Limit theorem, Mathematics of Operations Research 21 (1996), 237-251.

[6] B. De Meyer and A. Marino, Duality and optimal strategies in the finitely repeated zero-sum games with incomplete information on both sides, Preprint, Hal-00193996, 2005.

[7] M. Heuer, Asymptotically optimal strategies in repeated games with incomplete information, International Journal of Game Theory 20 (1992), 377-392.

[8] J.-F. Mertens and S. Zamir, The value of two-person zero-sum repeated games with lack of information on both sides, International Journal of Game Theory 1 (1971), 39-64.

[9] M. Oliu-Barton, Differential games with asymmetric and correlated information, Preprint. (2013).

[10] R.T. Rockafellar, Convex Analysis, Princeton Landmarks in Mathematics and Physics (Princeton University Press, Princeton, NJ), 1997.

[11] S. Sorin, A First Course on Zero-Sum Repeated Games, Springer, 2002. 